\title{
Evaluation of the trachea and bronchi for infiltration and ruptures of a neoplastic process originating from the thyroid gland
}

\author{
Mitko Mitev, MD \\ Department of Medical Physics, Biophysics, Roentgenology and Radiology, Faculty of Medicine, \\ Department of Diagnostic Imaging, University Hospital 'St. Kirkovich', \\ Trakia University - Stara Zagora, 6000 Stara Zagora, Bulgaria
}

\begin{abstract}
Background: evaluation of the trachea and bronchi for infiltration and ruptures of a neoplastic process originating from the thyroid gland.

Methods: virtual bronchoscopy with multidetector computed tomography; standard criteria.

Results: The present is a case of changes in the shape of the trachea from external compression, stenosis and dislocation, diagnosed with virtual bronchoscopy in a woman of 57 years, as a result of a tumour formation, originating from the thyroid gland (struma nodosa). The described morphological characteristics of the findings from the axial and multiplanar reconstructions of the native and intravenous postcontrast computed tomography point to the benign characteristics of the formation

Conclusions: with virtual bronchoscopy, the degree of stenosis and its length can be established, a lack of infiltration of the wall by the formation can be proved, as well as the absence of enlarged lymph nodes. Virtual bronchoscopy allows making evaluation of the carina. VB of the CT examination did not detect infiltrations and abnormalities of the wall as fistulous passages, and no evidence of tracheal and bronchial ruptures from the described neoplastic process.

Keywords: Virtual bronchoscopy, Thyroid carcinoma, Tracheal rupture
\end{abstract}

\author{
Grant support: No \\ Conflict of interest: No \\ List of abbreviations \\ CT: Computed tomography \\ FB: Fiberoptic bronchoscopy \\ HE: Hounsfield units \\ MDCT: Multidetector computed tomography \\ MPR: Multiplanar reconstructions \\ VB: Virtual bronchoscopy
}

Running head: virtual bronchoscopy thyroid gland neoplasm

\section{Introduction}

Virtual bronchoscopy (VB) is a new non-invasive method for three-dimensional reconstruction and evaluation of the tracheobronchial tree $(1,2,3)$. It involves the application of three-dimensional techniques for the imaging of the respiratory passages, allowing the simulation and navigation of real bronchoscopic procedures such as the detection of endobronchial abnormalities; performing transbronchial needle aspiration; diagnosis of small peripheral lung lesions $(<20 \mathrm{~mm}$ in diameter $)$, etc. (4). Multidetector computed tomography is the appropriate modality for examining suspected respiratory pathologies $(5,6,7)$. The present study aims to present the potential of $\mathrm{VB}$ in ruptures and stenoses of the trachea as a result of a benign tumour formation originating from the thyroid gland.

\section{Methods}

The object of the study is a 57 years old woman with changes in the shape of the trachea from external compression, stenosis and dislocation, as a result of a tumour formation of the thyroid gland. She is diagnosed with the modality of VB. The study was performed on 64 MDCT "Siemens Definition AS". Scanning was performed with post-processing and was done at $0.6 \mathrm{~mm}$. MDVB was performed based on the obtained 
reconstructed images. The additional processing was executed with a protocol for virtual bronchoscopy. "Siemens" workstation was used with "Singovia VB20" software, and "Siemens Definition AS" workstations with the capacity to track and match the images in the axial, coronary, sagittal planes. Multiplanar reconstructions were performed by applying MIP techniques and capabilities to archive and export images and video. The optimized standard protocol was used (current strength $80-100 \mathrm{mAs}$, voltage $100 \mathrm{kV}, 3$ $\mathrm{mm}$ beam collimation, reconstruction of $0.6 \mathrm{~mm}$, rotation speed at $0.5 \mathrm{sec}$, pitch D-FOV Large). Criteria for pathological changes in ruptures of trachea include localization of thyroid carcinoma, disposition of carcinoma; length of stenosis ( $\mathrm{mm}$ ); a distance of the carina ( $\mathrm{mm})$.

\section{Results}

The present is a case of changes in the shape of the trachea from external compression, stenosis and dislocation, diagnosed with VB in a woman of 57 years, as a result of a tumour formation, originating from the thyroid gland (struma nodosa). On the right, in the lower cervical region, originating from the right thyroid lobe of the thyroid gland, $4 \mathrm{~cm}$ above the carina, a large oval heterodense formation with the density of 42-56 Hounsfield units (HE) is visible with native CT scan, as well as the presence of irregular macrocalcifications. It passes through the upper thoracic aperture and enters the upper posterior mediastinum. The outlines of the formation appear correct, smooth and sharp. The dimensions of the findings in the axial plane are $66.1 / 53.0 \mathrm{~mm}$, and in the coronary plane $-49.1 / 45.4 \mathrm{~mm}$. On the native CT scan, the described findings are located in the upper posterior mediastinum and without clear distinction from the adjoining vena cava superior, arteria subclavia dextra, truncus carotis dextra, and also from the distal part of the trachea and the right upper lobe bronchus. Compression and dislocation to the left, distal to the trachea, and stenosis of the lumen up to $9.0 \mathrm{~mm}$ are found. There is an arcuate compression caudal to the right upper lobe bronchus with partial, about 20 percent stenosis of the bronchus. The carina is unchanged and with a trade angle. After intravenous administration of contrast medium (Yopamiro, 350-100 ml), strong and inhomogeneous contrast medium capturing is seen as the findings increased its density to 99.7 HE. The adjacent vascular structures are depicted normally, with smooth and sharp outlines, partially stenosed and compressed, with their walls well-shaped and intact. The trachea and adjacent bronchus appeare to be compressed without being infiltrated by the findings.

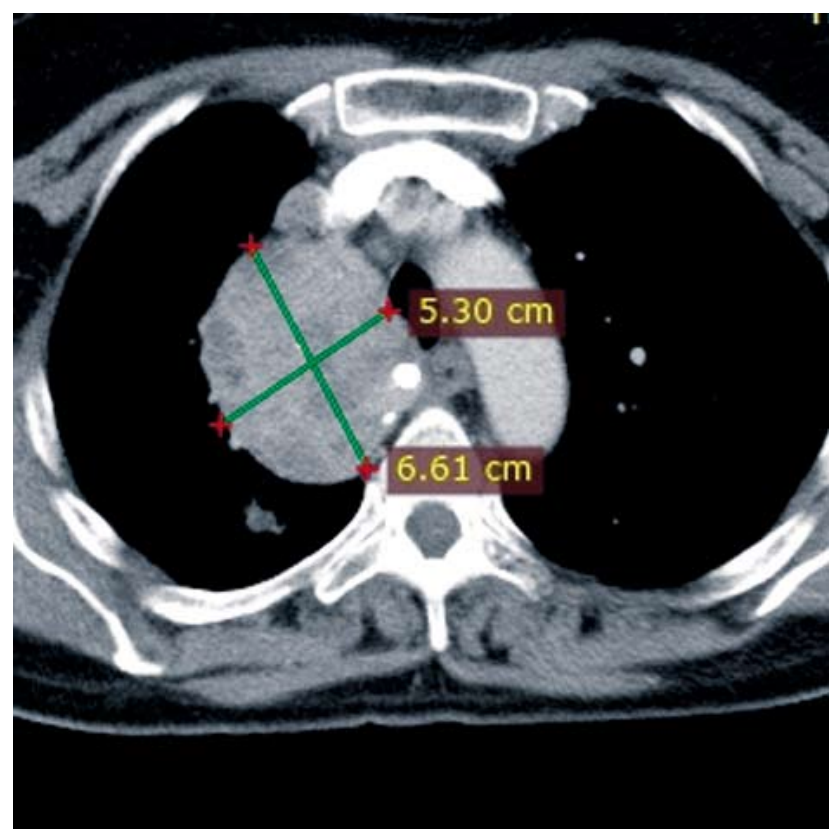

Figure 1. Tumor in axial projection with contrast medium (CT )

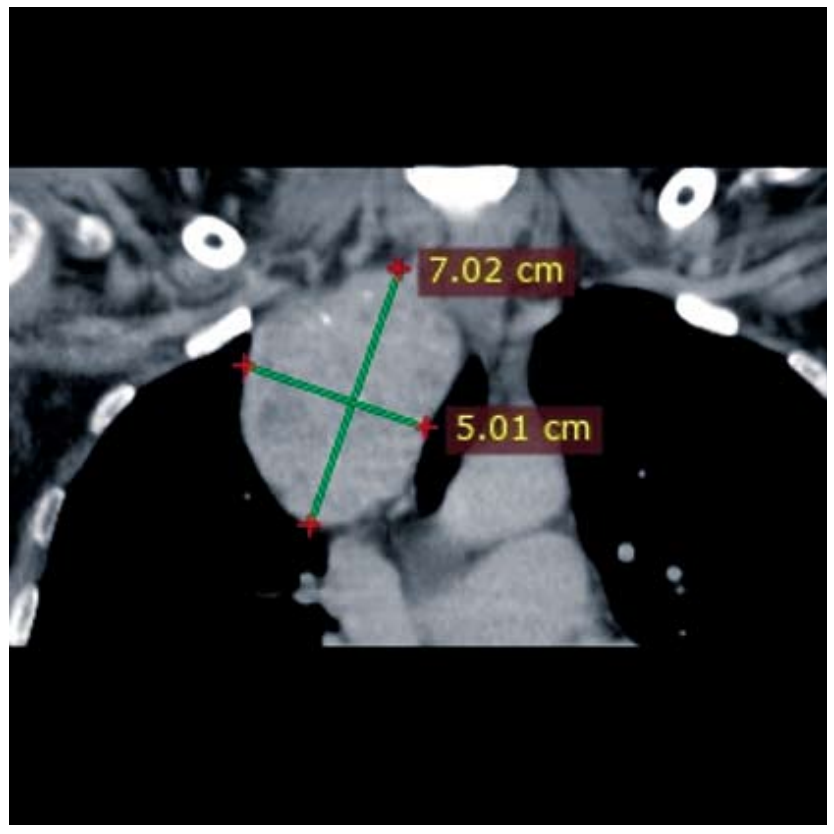

Figure 2. Tumor in the coronary plane (CT) 


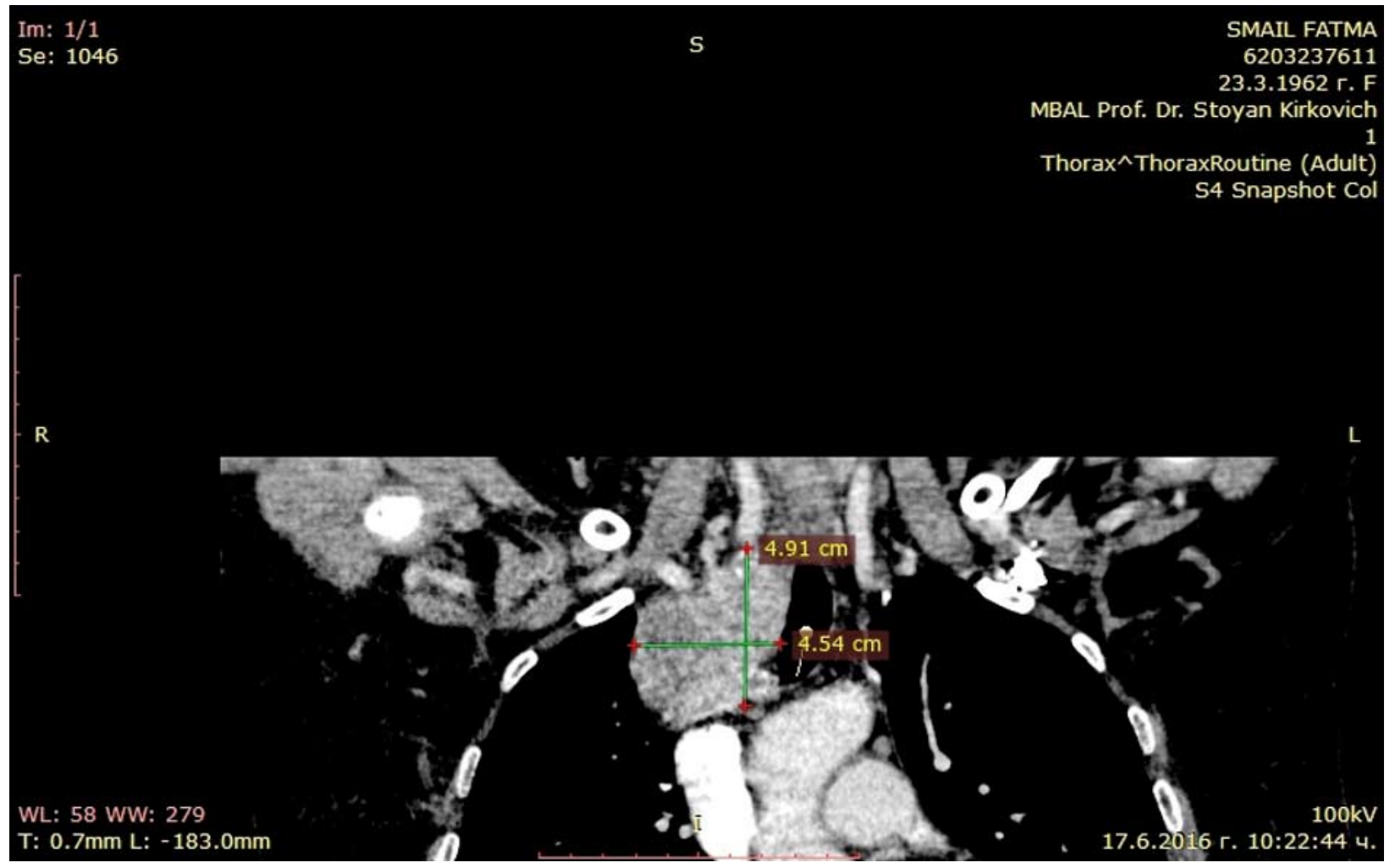

Figure 3. Coronary plane with intravenous contrast (CT)

On the made VB, the lumen of the trachea was arch-compressed on the right side, for a length of $50 \mathrm{~mm}$, narrowing it laterally to $9.0 \mathrm{~mm}$. The wall had a regular arcuate linear impression from external compression. It looks with smooth and straight outline without disturbed integrity. No visible signs of tracheal and bronchial infiltration can be seen from the described finding. Distally the segmental and subsegmental bronchi are also with preserved passability. No pathological changes are seen on the left. The entire mediastinum is located to the left. Significantly enlarged lymph nodes are not seen.

The described morphological characteristics of the findings from the axial and multiplanar reconstructions of the native and intravenous post-contrast computed tomography (CT) point to the benign characteristics of the formation (struma nodosa), which originates from the right thyroid lobe and enters the upper posterior mediastinum, before the upper thoracic aperture, compressing and dislocating the vessels, trachea and bronchi without infiltrating them.

The post-processing VB also proves regular and smooth outline of the wall of the trachea and bronchi, with narrowing of the lumen, regular linearity of the contours, preserved integrity of these organs, without evidence of infiltration from the pathological findings.

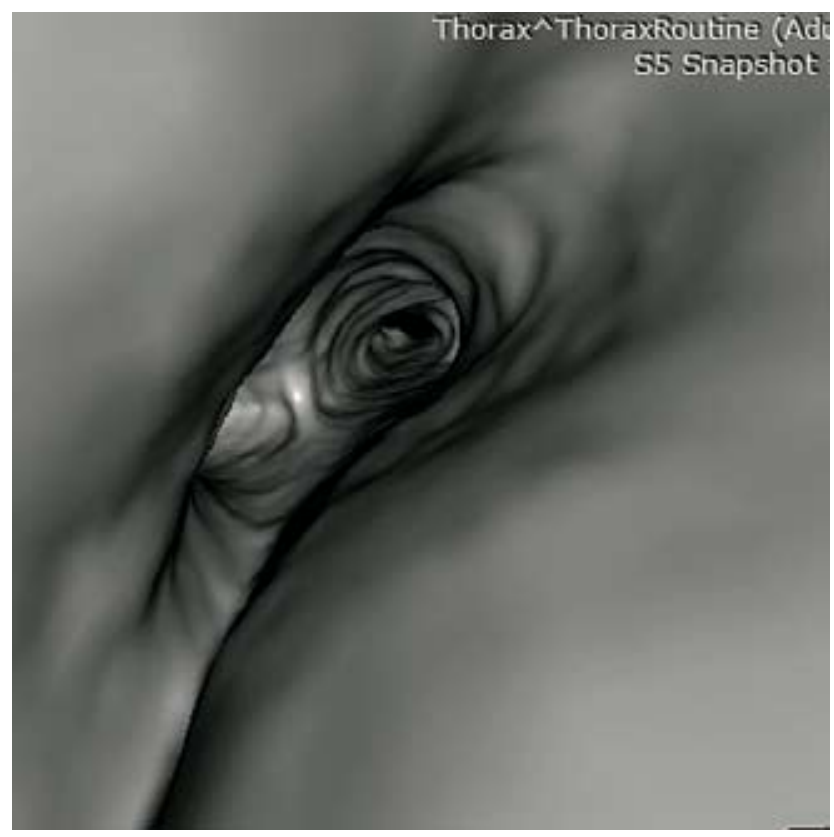

Figure 4. Tracheal stenosis and right upper bronchial bronchus (VB) 


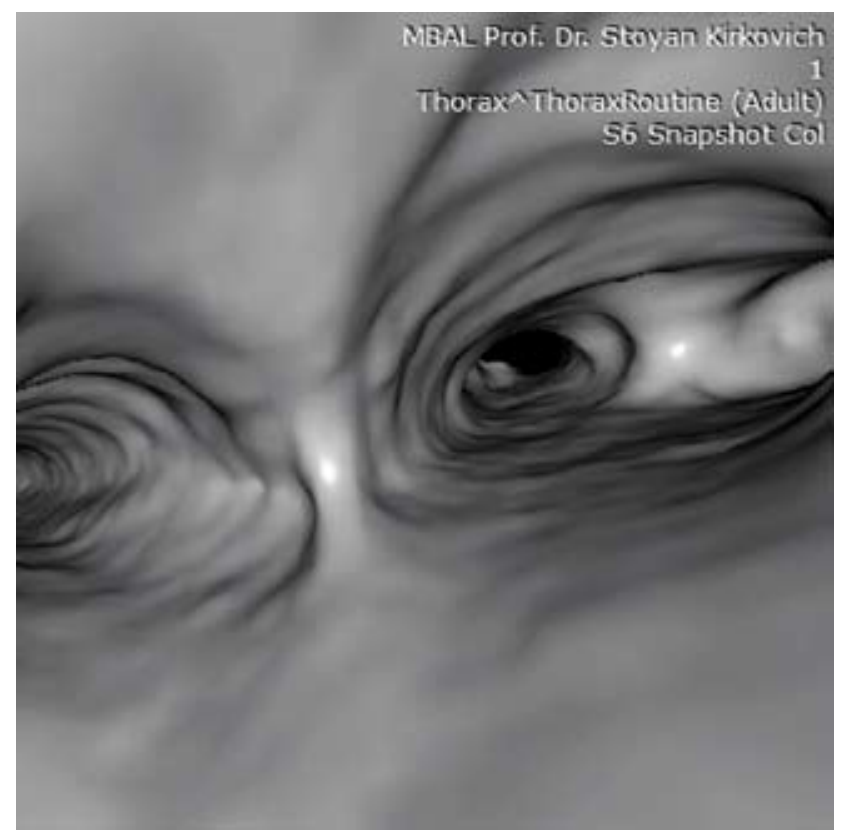

Figure 5. Arcuate compression and stenosis of the trachea (VB)

\section{Discussions}

Shepard et al. (8) provide an overview of the possibilities of depicting various benign and malignant diseases that directly or indirectly affect the trachea, including cancers of the thyroid. They identify CT as an appropriate modality for their imaging and for improving diagnosis when evaluating tracheal abnormalities. CT has several advantages such as excellent spatial resolution and the ability to generate multiplanar reformations and $3 \mathrm{D}$ reconstructions; rapid and non-invasive evaluation of the tracheobronchial tree. CT detectors retain the spatial resolution in the axial, coronal and sagittal planes. Multidetector computed tomography (MDCT) has been identified as an excellent diagnostic tool for detecting, localizing, and examining the extent of respiratory pathologies, regardless of whether the disease is local or remotely situated. VB 3D reconstruction from CT images is a useful diagnostic tool because it offers a non-invasive technique for examining the tracheobronchial tree $(5,6,7)$. Ferretti et al. (9) investigate the use of VB in imaging stenoses of the tracheobronchial tree. The study is conducted with 29 patients, with 41 stenoses proven with FB. Two of the stenoses they report as a result of Malignant Thyroid Carcinoma. The remaining stenoses are the result of Lung cancer (27); Esophageal carcinoma (1); Benign diseases (11). They achieve good imaging qual- ity of the airway inner walls in 27 of 29 patients (93\%). They find 39 of 41 airway stenoses (95\%). Through MPR and VB, they do not detect only two small stenoses $(<25 \%)$ in patients in the very bad condition who do not meet the breathing retention requirements during the one-minute hyperventilation period before $\mathrm{CT}$ scanning. The authors also indicate that all stenoses depicted by VB are also detected by axial CT scan. According to the authors, the assessment of stenosis length and airway wall thickness at the stenosis level requires the simultaneous display of the multiplanar reconstruction (MPR) and VB images. They do not detect mucosal abnormalities with VB. They recommend that VB images be combined with axial images or MPR. In their opinion the advantage of VB is the fact that the perspective of VB on display is within the axis of the airways, thus explaining the more prominent results of $\mathrm{CT}$ with $\mathrm{VB}$ than with axial $\mathrm{CT}$ or even with MPR alone. Finkelstein et al. (1) apply the VB method to compare its diagnostic potential to that of FB in assessing suspected tracheobronchial lesions in patients with thoracic malignant tumours. Thirty-two patients are studied, 12 of them with non-small-cell lung cancer; 3 with small cell lung cancer; 8 with renal cell cancer; 5 with metastatic melanoma; 2 with metastatic thyroid cancer; 1 with sarcoma and 1 with oesophagal cancer. Using VB, they identify 18 of the 22 anomalies identified by FB. These are 13 of 13 obstructive lesions; 5 of 6 endoluminal lesions; 0 of 3 mucosal lesions. They determine $100 \%$ sensitivity of the method for the diagnosis of obstructive lesions; $83 \%$ for endoluminal lesions; $0 \%$ for mucosal lesions and $82 \%$ for all other abnormalities. According to the authors, the specificity of VB is $100 \%$. The authors conclude that VB is a promising non-invasive modality for the identification of bronchial obstruction and endoluminal lesions, as well as for the evaluation of the tracheobronchial tree outside the stenoses. Through VB, the authors find seven obstructive lesions not visualized through FB. The reasons for this, according to the authors, are the limitation of the size of the bronchoscope ( 5 patients) and the location of the lesions outside the stenoses (2 patients). They point out that VB does not allow the detection of thin mucosal lesions and therefore, in their view, this modality may not be suitable for identifying premalignant lesions in the airways. The authors indicate that VB is a necessary method as a source of additional information that may 
be useful in the management of malignant lung tumours. Unverdi et al. (10) compare MDCT VB and $\mathrm{FB}$ when evaluating tracheobronchial lesions. Forty-four patients are studied. They view FB as the "gold" standard for evaluating the results. They find $92 \%$ sensitivity; $73 \%$ specificity, $84 \%$ precision of the VB method, etc. Malignant infiltration and extraluminal compression are not visualized by VB $(1,11)$. VB delineates the tracheobronchial anatomy beyond high-grade stenoses but does not visualize mucosal lesions $(1,12)$.
With VB, the degree of stenosis and its length can be established, a lack of infiltration of the wall by the formation can be proved, as well as the absence of enlarged lymph nodes. VB allows making an evaluation of the carina. VB of the CT examination did not detect infiltrations and abnormalities of the wall as fistulous passages, and no evidence of tracheal and bronchial ruptures from the described neoplastic process.

\title{
References
}

1. Finkelstein S E, Summers R M, Nguyen D M, Stewart IV J H, Tretler J A, Schrump D S. Virtual bronchoscopy for evaluation of malignant tumors of the thorax. J Thorac Cardiovasc Surg 2002; 123: 967-7. doi:10.1067/mtc.2002.121495

2. Obretenov E, Arabadzhiev G, Vylcheva S. Postintubation Thrachea Lesion - A Modern Behavior Algorithm. J Bulg Thor Card and Vasc Surg 2014; $1: 15-23$

3. Mitev M A. Virtual bronchoscopy with Multidetector computer tomography. PhD Thesis, Stara Zagora: Trakia University, $2017: 180$.

4. Shinagava N, Yamazaki K, Onodera Y, Asano F, Ishida T, Moriya H, Nishimur M. Virtual bronchoscopic navigation system shortens the examination time - Feasibility study of virtual bronchoscopic navigation system. Lung Cancer 2007; 56: 201-206. Doi: 10.1016/ j.lungcan.2006.12.005

5. Boiselle Ph, Lee K, Ernst A. Multidetector CT of the Central Airways. Journal of Thoracic Imaging 2005; 20 (3): 186-195. DOI: 10.1097/01. rti.0000171624.84951.f2, PMID:16077334

6. Kang E. Large Airway Diseases. Journal of Thoracic Imaging 2011; 26 (4): 249-262. DOI: 10.1097/RTI.0b013e31822428ef, PMID:22009079

7. Bedayat A, Yang E, Chandili S, Galera P, Chalian H, Ansari-Gilani K, Guo H H. Tracheobronchial Tumors: Radiologic - Pathologis Correlation of Tumors and Mimics. Current Problems in Diagnostic Radiology 2019; 1-10. https://doi.org/10.1067/j.cpradiol.2019.04.003

8. Shepard J O, Flores E J, Abbott G F. Imaging of the trachea. Ann Cardiothorac Surg 2018; 7 (2): 197-209. doi: 10.21037/acs.2018.03.09

9. Ferretti G R, Knoplioch J, Bricault I, Brambilla Ch, Coulomb M. Central airway stenoses: preliminary results of spiral - CT - generated virtual bronchoscopy simulations in 29 patients. Eur. Radiol. 1997; 7: 854-859. https://doi.org/10.1007/s003300050218

10. Unverdi Z, Kervancioglu R, Unverdi S, Menzilcioglu M S. In the evaluation of tracheobronchial lesions, MDCT virtual bronchoscopy with fiber optic bronchoscopy comparison. Medical Science and Discovery 2019; 6 (8): 136-44. Doi: 10.17546/msd.584332

11. Fleiter T, Merkle E M, Aschoff A J, et al. Comparison of real-time virtual andfiberoptic bronchoscopy in patients with bronchial carcinoma: opportunities and limitations. Am J Roentgenol 1997; 169: 1591-1595. DOI: 10.2214/ajr.169.6.9393172 PMID: 9393172

12. Liewald F, Lang G, Fleiter T H, Sokiranski R, Halter G, Orend K H. Comparison of virtual and fiberoptic bronchoscopy.Thorac Cardio-vasc Surg 1998; 46: 361-364. Doi: 10.1055/s-2007-1010254

\author{
Correspondence: \\ D-r Mitko Mitev, MD
}

Department of Medical Physics, Biophysics, Roentgenology and Radiology, Faculty of Medicine, Department of Diagnostic Imaging, University Hospital 'St. Kirkovich',

Trakia University - Stara Zagora,

6000 Stara Zagora, Bulgaria;

E-mail:mitev.mitko69@gmail.com

Mobile: +359887 706079 